\title{
Critical points, the Gauss curvature equation and Blaschke products
}

\author{
Daniela Kraus and Oliver Roth
}

\begin{abstract}
In this survey paper we discuss the problem of characterizing the critical sets of bounded analytic functions in the unit disk of the complex plane. This problem is closely related to the Berger-Nirenberg problem in differential geometry as well as to the problem of describing the zero sets of functions in Bergman spaces. It turns out that for any non-constant bounded analytic function in the unit disk there is always a (essentially) unique "maximal" Blaschke product with the same critical points. These maximal Blaschke products have remarkable properties simliar to those of Bergman space inner functions and they provide a natural generalization of the class of finite Blaschke products.
\end{abstract}

\section{Critical points of bounded analytic functions}

A sequence of points $\left(z_{j}\right)$ in a subdomain $\Omega$ of the complex plane $\mathbb{C}$ is called the zero set of an analytic function $f: \Omega \rightarrow \mathbb{C}$, if $f$ vanishes precisely on this set. This means that if the point $\xi \in \Omega$ occurs $m$ times in the sequence, then $f$ has a zero at $\xi$ of precise order $m$, and $f(z) \neq 0$ for $z \in \Omega \backslash\left(z_{j}\right)$. The following classical theorem due to Jensen [32, Blaschke [7] and F. and R. Nevanlinna [54] characterizes completely the zero sets of bounded analytic functions defined in the open unit disk $\mathbb{D}:=\{z \in \mathbb{C}:|z|<1\}$.

Theorem A. Let $\left(z_{j}\right)$ be a sequence in $\mathbb{D}$. Then the following statements are equivalent.

(a) There is an analytic self-map of $\mathbb{D}$ with zero set $\left(z_{j}\right)$.

(b) There is a Blaschke product with zero set $\left(z_{j}\right)$.

(c) The sequence $\left(z_{j}\right)$ fulfills the Blaschke condition, i.e. $\sum_{j=1}^{\infty}\left(1-\left|z_{j}\right|\right)<+\infty$.

We call a sequence of points $\left(z_{j}\right)$ in a domain $\Omega \subseteq \mathbb{C}$ the critical set of an analytic function $f: \Omega \rightarrow \mathbb{C}$, if $\left(z_{j}\right)$ is the zero set of the first derivative $f^{\prime}$ of the function $f$. There is an extensive literature on critical sets. In particular, there are many interesting results on the relation between the zeros and the critical points of analytic and harmonic functions. A classical reference for all this is the book of Walsh 65.

1991 Mathematics Subject Classification. 30H05, 30J10, 35J60, 30H20, 30F45, 53A30.

Key words and phrases. Blaschke products, Elliptic PDEs, Bergman spaces.

Supported by the Deutsche Forschungsgemeinschaft (Grants: Ro 3462/3-1 and Ro 3462/3-2). 
The first aim of this survey paper is to point out the following analogue of Theorem $\mathrm{A}$ for the critical sets of bounded analytic functions instead of their zeros sets.

TheOREM 1.1. Let $\left(z_{j}\right)$ be a sequence in $\mathbb{D}$. Then the following statements are equivalent.

(a) There is an analytic self-map of $\mathbb{D}$ with critical set $\left(z_{j}\right)$.

(b) There is a Blaschke product with critical set $\left(z_{j}\right)$.

(c) There is a function in the weighted Bergman space

$$
\mathcal{A}_{1}^{2}=\left\{g: \mathbb{D} \rightarrow \mathbb{C} \text { analytic }: \iint_{\mathbb{D}}\left(1-|z|^{2}\right)|g(z)|^{2} d \sigma_{z}<+\infty\right\}
$$

with zero set $\left(z_{j}\right)$.

Here, and throughout, $\sigma_{z}$ denotes two-dimensional Lebesgue measure with respect to $z$.

For the special case of finite sequences, a result related to Theorem $1.1 \mathrm{can}$ be found in work of Heins [27, §29], Wang \& Peng [66, Zakeri [70 and Stephenson 62. They proved that for every finite sequence $\left(z_{j}\right)$ in $\mathbb{D}$ there is always a finite Blaschke product whose critical set coincides with $\left(z_{j}\right)$, see also Remark 2.10 below. A recent generalization of this result to infinite sequences is discussed in [42]. There it is shown that every Blaschke sequence $\left(z_{j}\right)$ is the critical set of an infinite Blaschke product. However, the converse to this, known as the Bloch-Nevanlinna conjecture [14, is false. According to a result of Frostman, there do exist Blaschke products whose critical sets fail to satisfy the Blaschke condition, see [13, Theorem 3.6]. Thus the critical sets of bounded analytic functions are not just the Blaschke sequences and the situation for critical sets is much more complicated than for zero sets.

Theorem1.1identifies the critical sets of bounded analytic functions as the zeros sets of functions in the Bergman space $\mathcal{A}_{1}^{2}$. The simple geometric characterization of the zero sets of bounded analytic functions via the Blaschke condition (c) in Theorem $\mathrm{A}$ has not found an explicit counterpart for critical sets yet. However, condition (c) of Theorem 1.1 might be seen as an implicit substitute. The zero sets of (weighted) Bergman space functions have intensively been studied in the 1970's and 1990's by Horowitz [29, 30, Korenblum [37, Seip [57, 58, Luecking 47. and many others. As a result quite sharp necessary conditions and sufficient conditions for the zero sets of Bergman space functions are available. In view of Theorem 1.1 all results about zero sets of Bergman space functions carry now over to the critical sets of bounded analytic functions and vice versa. Unfortunately, a geometric characterization of the zero sets of (weighted) Bergman space functions is still unknown, "and it is well known that this problem is very difficult", cf. [25, p. 133].

The implications "(b) $\Longrightarrow(\mathrm{a})$ " and "(a) $\Longrightarrow(\mathrm{c})$ " of Theorem 1.1 are easy to prove. In fact, the statement "(a) $\Longrightarrow$ (c)" follows directly from the LittlewoodPaley identity (see 60, p. 178]), which says that for any holomorphic function $f: \mathbb{D} \rightarrow \mathbb{D}$ the derivative $f^{\prime}$ belongs to $\mathcal{A}_{1}^{2}$. Hence the critical set of any nonconstant bounded analytic function is trivially the zero set of a function in $\mathcal{A}_{1}^{2}$. It is however not true that any $\mathcal{A}_{1}^{2}$ function is the derivative of a bounded analytic function, so "(c) $\Longrightarrow$ (a)" is more subtle. 
In the following sections, we describe some of the ingredients of the proofs of the implications "(a) $\Longrightarrow(\mathrm{b})$ " and "(c) $\Longrightarrow(\mathrm{a})$ " in Theorem 1.1 as well as some further results connected to it. In particular, we explain the close relation of Theorem 1.1 to conformal differential geometry and the solvability of the Gauss curvature equation. This paper is expository, so there are essentially no proofs. For the proofs we refer to $39,40,41,42,44$. Background material on Hardy spaces and Bergman spaces can be found e.g. in the excellent books $15,19,22,[25,36,48$.

Finally, we draw attention to the recent paper [20] of P. Ebenfelt, D. Khavinson and H.S. Shapiro and the references therein, where the difficult problem of constructing a finite Blaschke product by its critical values is discussed.

\section{Conformal metrics and maximal Blaschke products}

An essential characteristic of the proof of the implication "(a) $\Longrightarrow(b)$ " in Theorem 1.1 is the extensive use of negatively curved conformal pseudometrics. We give a short account of some of their properties and refer to $[\mathbf{5}, \mathbf{2 7}, \mathbf{3 5}, \mathbf{3 8}, \mathbf{4 3}, \mathbf{6 1}$ for more details. In the following, $G$ and $D$ always denote domains in the complex plane $\mathbb{C}$.

2.1. Conformal metrics and developing maps. We recall that a nonnegative upper semicontinuous function $\lambda$ on $G, \lambda: G \rightarrow[0,+\infty), \lambda \not \equiv 0$, is called conformal density on $G$. The corresponding quantity $\lambda(z)|d z|$ is called conformal pseudometric on $G$. If $\lambda(z)>0$ for all $z \in G$, we say $\lambda(z)|d z|$ is a conformal metric on $G$. We call a conformal pseudometric $\lambda(z)|d z|$ regular on $G$, if $\lambda$ is of class $C^{2}$ in $\{z \in G: \lambda(z)>0\}$.

ExAMPLE 2.1 (The hyperbolic metric). The ubiquitous example of a conformal metric is the Poincaré metric or hyperbolic metric

$$
\lambda_{\mathbb{D}}(z)|d z|:=\frac{|d z|}{1-|z|^{2}}
$$

for the unit disk $\mathbb{D}$. Clearly, $\lambda_{\mathbb{D}}(z)|d z|$ is a regular conformal metric on $\mathbb{D}$.

The (Gauss) curvature $\kappa_{\lambda}$ of a regular conformal pseudometric $\lambda(z)|d z|$ on $G$ is defined by

$$
\kappa_{\lambda}(z):=-\frac{\Delta(\log \lambda)(z)}{\lambda(z)^{2}}
$$

for all points $z \in G$ where $\lambda(z)>0$. Note that if $\lambda(z)|d z|$ is a regular conformal metric with curvature $\kappa_{\lambda}=\kappa$ on $G$, then the function $u:=\log \lambda$ satisfies the partial differential equation (Gauss curvature equation)

$$
\Delta u=-\kappa(z) e^{2 u}
$$

on $G$. If, conversely, a realvalued $C^{2}$-function $u$ is a solution of the Gauss curvature equation (2.1) on $G$, then $\lambda(z):=e^{u(z)}$ induces a regular conformal metric $e^{u(z)}|d z|$ on $G$ with curvature $\kappa$.

ExAmple 2.2. The hyperbolic metric $\lambda_{\mathbb{D}}(z)|d z|$ on $\mathbb{D}$ has constant negative curvature1 -4 .

\footnotetext{
${ }^{1}$ Warning: A rival school of thought calls $\frac{2|d z|}{1-|z|^{2}}$ the hyperbolic metric of $\mathbb{D}$. This metric has constant curvature -1 .
} 
Using analytic maps, conformal metrics can be transferred from one domain to another as follows. Let $\lambda(w)|d w|$ be a conformal pseudometric on a domain $D$ and let $w=f(z)$ be a non-constant analytic map from another domain $G$ to $D$. Then the conformal pseudometric

$$
\left(f^{*} \lambda\right)(z)|d z|:=\lambda(f(z))\left|f^{\prime}(z)\right||d z|,
$$

defined on $G$, is called the pullback of $\lambda(w)|d w|$ under the map $f$. Now, Gauss curvature is important since it is a conformal invariant in the following sense.

Theorem B (Theorema Egregium). For every analytic map $w=f(z)$ and every regular conformal pseudometric $\lambda(w)|d w|$ the relation

$$
\kappa_{f^{*} \lambda}(z)=\kappa_{\lambda}(f(z))
$$

is satisfied provided $\lambda(f(z))\left|f^{\prime}(z)\right|>0$.

Hence pullbacks of conformal pseudometrics can be used for constructing conformal pseudometrics with various prescribed properties while controlling their curvature. For instance, if $\lambda(w)|d w|$ is a conformal metric on $D$ (without zeros) and $w=f(z)$ is a non-constant analytic map from $G$ to $D$, then $\left(f^{*} \lambda\right)(z)|d z|$ is a pseudometric on $G$ with zeros exactly at the critical points of $f$. In order to take the multiplicity of the zeros into account, it is convenient to introduce the following formal definition.

Definition 2.3 (Zero set of a pseudometric). Let $\lambda(z)|d z|$ be a conformal pseudometric on $G$. We say $\lambda(z)|d z|$ has a zero of order $m_{0}>0$ at $z_{0} \in G$ if

$$
\lim _{z \rightarrow z_{0}} \frac{\lambda(z)}{\left|z-z_{0}\right|^{m_{0}}} \quad \text { exists and } \neq 0 .
$$

We will call a sequence $\mathcal{C}=\left(\xi_{j}\right) \subset G$

$$
\left(\xi_{j}\right):=(\underbrace{z_{1}, \ldots, z_{1}}_{m_{1}-\text { times }}, \underbrace{z_{2}, \ldots, z_{2}}_{m_{2} \text {-times }}, \ldots), z_{k} \neq z_{n} \text { if } k \neq n,
$$

the zero set of a conformal pseudometric $\lambda(z)|d z|$, if $\lambda(z)>0$ for $z \in G \backslash \mathcal{C}$ and if $\lambda(z)|d z|$ has a zero of order $m_{k} \in \mathbb{N}$ at $z_{k}$ for all $k$.

Now, every conformal pseudometric of constant curvature -4 can locally be represented by a holomorphic function. This is the content of the following result.

Theorem C (Liouville's Theorem). Let $\mathcal{C}$ be a sequence of points in a simply connected domain $G$ and let $\lambda(z)|d z|$ be a regular conformal pseudometric on $G$ with constant curvature -4 on $G$ and zero set $\mathcal{C}$. Then $\lambda(z)|d z|$ is the pullback of the hyperbolic metric $\lambda_{\mathbb{D}}(z)|d z|$ under some analytic map $f: G \rightarrow \mathbb{D}$, i.e.

$$
\lambda(z)=\frac{\left|f^{\prime}(z)\right|}{1-|f(z)|^{2}}, \quad z \in G .
$$

If $g: G \rightarrow \mathbb{D}$ is another analytic function, then $\lambda(z)=\left(g^{*} \lambda_{\mathbb{D}}\right)(z)$ for $z \in G$ if and only if $g=T \circ f$ for some conformal automorphism $T$ of $\mathbb{D}$.

A holomorphic function $f$ with property (2.2) will be called a developing map for $\lambda(z)|d z|$. Note that the critical set of each developing map coincides with the zero set of the corresponding conformal pseudometric. 
EXAMPLE 2.4. The developing maps of the hyperbolic metric $\lambda_{\mathbb{D}}(z)|d z|$ are precisely the conformal automorphisms of $\mathbb{D}$, i.e., the finite Blaschke products of degree 1.

For later applications we wish to mention the following variant of Liouville's Theorem.

Theorem 2.5. Let $G$ be a simply connected domain and let $h: G \rightarrow \mathbb{C}$ be an analytic map. If $\lambda(z)|d z|$ is a regular conformal metric with curvature $-4|h(z)|^{2}$, then there exists a holomorphic function $f: G \rightarrow \mathbb{D}$ such that

$$
\lambda(z)=\frac{1}{|h(z)|} \frac{\left|f^{\prime}(z)\right|}{1-|f(z)|^{2}}, \quad z \in G .
$$

Moreover, $f$ is uniquely determined up to postcomposition with a unit disk automorphism.

Liouville [46] stated Theorem $\mathrm{C}$ for the special case that $\lambda(z)|d z|$ is a regular conformal metric. We therefore refer to Theorem $\mathrm{C}$ as well as to Theorem 2.5 as Liouville's theorem. Theorem $[\mathrm{C}$ and in particular the special case that $\lambda(z)|d z|$ is a conformal metric has a number of different proofs, see for instance $[\mathbf{8}, \mathbf{1 1}, \mathbf{1 2}$, 50, 56, 67. Theorem 2.5 is discussed in 42.

Liouville's theorem plays an important rôle in this paper. It provides a bridge between the world of bounded analytic functions and the world of conformal pseudometrics with constant negative curvature. The critical points on the one side correspond to the zeros on the other side. Unfortunately, this bridge only works for simply connected domains, see Remark 4.9.

2.2. Maximal conformal pseudometrics and maximal Blaschke products. Apart from having constant negative curvature the hyperbolic metric on $\mathbb{D}$ has another important property: it is maximal among all regular conformal pseudometrics on $\mathbb{D}$ with curvature bounded above by -4 . This is the content of the following result.

TheOREM D (Fundamental Theorem). Let $\lambda(z)|d z|$ be a regular conformal pseudometric on $\mathbb{D}$ with curvature bounded above by -4 . Then $\lambda(z) \leq \lambda_{\mathbb{D}}(z)$ for every $z \in \mathbb{D}$.

Theorem $\mathrm{D}$ is due to Ahlfors [1] and it is usually called Ahlfors' lemma. However, in view of its relevance Beardon and Minda proposed to call Ahlfors' lemma the fundamental theorem. We will follow their suggestion in this paper. As a result of the fundamental theorem we have

$$
\begin{array}{r}
\lambda_{\mathbb{D}}(z)=\max \{\lambda(z): \lambda(z)|d z| \text { is a regular conformal pseudometric on } \mathbb{D} \\
\text { with curvature } \leq-4\}
\end{array}
$$

for any $z \in \mathbb{D}$.

REMARK 2.6 (Developing maps and universal coverings). Let $G$ be a hyperbolic subdomain of the complex plane $\mathbb{C}$, i.e., the complement $\mathbb{C} \backslash G$ consists of more than one point. In analogy with the Poincaré metric for the unit disk, a regular conformal metric $\lambda_{G}(z)|d z|$ of constant curvature -4 on $G$ is said to be the hyperbolic metric for $G$, if it is the maximal regular conformal pseudometric with curvature $\leq-4$ on $G$, i.e. $\lambda(z) \leq \lambda_{G}(z), z \in G$, for all regular conformal pseudometrics $\lambda(z)|d z|$ on $G$ with curvature bounded above by -4 . Then $\lambda_{G}(z)|d z|$ 
and $\lambda_{\mathbb{D}}(z)|d z|$ are connected via the universal coverings $\pi: \mathbb{D} \rightarrow G$ of $G$ by the formula $\left(\pi^{*} \lambda_{G}\right)(z)|d z|=\lambda_{\mathbb{D}}(z)|d z|$, see for example [27, $\left.\S 9\right]$ and [43, 49]. Hence, every branch of the inverse of a universal covering map $\pi: \mathbb{D} \rightarrow G$ is locally the developing map of the hyperbolic metric $\lambda_{G}(z)|d z|$ of $G$. In particular, if $G$ is a hyperbolic simply connected domain, then the developing maps for $\lambda_{G}(z)|d z|$ are precisely the conformal mappings from $G$ onto $\mathbb{D}$.

We now consider prescribed zeros.

Theorem E. Let $\mathcal{C}=\left(\xi_{j}\right)$ be a sequence of points in $G$ and

$\Phi_{\mathcal{C}}:=\{\lambda: \lambda(z)|d z|$ is a regular conformal pseudometric in $G$ with curvature $\leq-4$ and zero set $\left.\mathcal{C}^{*} \supseteq \mathcal{C}\right\}$.

If $\Phi_{\mathcal{C}} \neq \emptyset$, then

$$
\lambda_{\max }(z):=\sup \left\{\lambda(z): \lambda \in \Phi_{\mathcal{C}}\right\}, \quad z \in G,
$$

induces the unique maximal regular conformal pseudometric $\lambda_{\max }(z)|d z|$ on $G$ with constant curvature -4 and zero set $\mathcal{C}$.

Thus, if $\Phi_{\mathcal{C}} \neq \emptyset$, i.e., if there exists at least one regular conformal pseudometric $\lambda(z)|d z|$ on $G$ with curvature $\leq-4$ whose zero set contains the given sequence $\mathcal{C}$, then there exists a (maximal) regular conformal pseudometric $\lambda(z)|d z|$ on $G$ with constant curvature -4 whose zero set is exactly the sequence $\mathcal{C}$. In particular, Theorem E can be applied, if there exists a non-constant holomorphic function $f: G \rightarrow \mathbb{D}$ with critical set $\mathcal{C}^{*} \supseteq \mathcal{C}$ since then the pseudometric $\left(f^{*} \lambda_{\mathbb{D}}\right)(z)|d z|$ belongs to $\Phi_{\mathcal{C}}$. The proof of Theorem Erelies on a modification of Perron's method and can be found in [27, $\S 12 \& \S 13]$ and [40.

EXAMPLE 2.7. If $G$ is a hyperbolic domain and $\mathcal{C}=\emptyset$, then the maximal regular conformal pseudometric $\lambda_{\max }(z)|d z|$ on $G$ with constant curvature -4 and zero set $\mathcal{C}$ is exactly the hyperbolic metric $\lambda_{G}(z)|d z|$ for $G$.

Thus maximal pseudometrics are generalizations of the hyperbolic metric and their developing maps are therefore of special interest.

Definition 2.8 (Maximal functions). Let $\mathcal{C}$ be a sequence of points in $\mathbb{D}$ such that there exists a maximal regular conformal pseudometric $\lambda_{\max }(z)|d z|$ on $\mathbb{D}$ with constant curvature -4 and zero set $\mathcal{C}$. Then every developing map for $\lambda_{\max }(z)|d z|$ is called maximal function for $\mathcal{C}$.

Some remarks are in order. First, in view of TheoremClevery maximal function is uniquely determined by its critical set $\mathcal{C}$ up to postcomposition with a unit disk automorphism and, conversely, the postcomposition of any maximal function with a unit disk automorphism is again a maximal function. Second, if $\mathcal{C}=\emptyset$, then the maximal functions for $\mathcal{C}$ are precisely the unit disk automorphisms, i.e., the finite Blaschke products of degree 1.

Now, we have the following result, see $[\mathbf{4 0}$.

THEOREM 2.9. Every maximal function is a Blaschke product.

It is to emphasize that since the postcomposition of any maximal function with a unit disk automorphism is again a maximal function, every maximal function is an indestructible Blaschke product. 
We note that Theorem E combined with Theorem 2.9 immediately gives the implication "(a) $\Longrightarrow$ (b)" in Theorem 1.1. In fact, if $f$ is a non-constant analytic self-map of $\mathbb{D}$ with critical set $\mathcal{C}$, then $\lambda(z)|d z|:=\left(f^{*} \lambda_{\mathbb{D}}\right)(z)|d z|$ is a regular conformal pseudometric on $\mathbb{D}$ with zero set $\mathcal{C}$. Thus Theorem $\mathbb{E}$ guarantees the existence of a maximal conformal pseudometric on $\mathbb{D}$ with zero set $\mathcal{C}$. Now, Theorem 2.9 says that the corresponding maximal function for $\mathcal{C}$ is a Blaschke product.

In the special case that the maximal function has finitely many critical points, the statement of Theorem 2.9 follows from the next result which is due to Heins 27. §29].

Theorem $\mathrm{F}$. Let $\mathcal{C}=\left(z_{1}, \ldots, z_{n}\right)$ be a finite sequence in $\mathbb{D}$ and $f: \mathbb{D} \rightarrow \mathbb{D}$ analytic. Then the following statements are equivalent.

(a) $f$ is a maximal function for $\mathcal{C}$.

(b) $f$ is Blaschke product of degree $n+1$ with critical set $\mathcal{C}$.

We shall give a quick proof of Theorem $\mathrm{F}$ in Remark 3.11 below.

REMARK 2.10 (Constructing finite Blaschke products with prescribed critical points). In his proof of Theorem $\mathrm{F}$. Heins showed that for any finite sequence $\mathcal{C}=\left(z_{1}, \ldots, z_{n}\right)$ in $\mathbb{D}$ there is always a finite Blaschke product $B$ of degree $n+1$ with critical set $\mathcal{C}$. The essential step is nonconstructive and consists in showing that the set of critical points of all finite Blaschke products of degree $n+1$, which is clearly closed, is also open in the poly disk $\mathbb{D}^{n}$ by applying Brouwer's fixed point theorem. The same result was later obtained by Wang \& Peng [66] and Zakeri [70 by using similar arguments. A completely different approach via Circle Packing is due to Stephenson, see Lemma 13.7 and Theorem 21.1 in [62]. Stephenson builds discrete finite Blaschke products with prescribed branch set and shows that under refinement these discrete Blaschke products converge locally uniformly in $\mathbb{D}$ to the desired classical Blaschke product.

We are not aware of any efficient constructive method for computing a (nondiscrete) finite Blaschke product from its critical points.

Maximal functions form a particular class of Blaschke products. It is therefore convenient to make the following definition.

Definition 2.11 (Maximal Blaschke products). A non-constant Blaschke product is called a maximal Blaschke product, if it is a maximal function for its critical set.

As was mentioned earlier, every maximal Blaschke product is indestructible and every finite Blaschke product is a maximal Blaschke product. Moreover, if $\mathcal{C}$ is the critical set of any non-constant analytic function $f: \mathbb{D} \rightarrow \mathbb{D}$, then there is maximal Blaschke product with critical set $\mathcal{C}$. A maximal Blaschke product is uniquely determined by its critical set $\mathcal{C}$ up to postcomposition with a unit disk automorphism.

Geometrically, the finite maximal Blaschke products are just the finite branched coverings of $\mathbb{D}$. One is therefore inclined to consider maximal Blaschke products for infinite branch sets as "infinite branched coverings": 


\begin{tabular}{|l|l|l|}
\hline critical set & maximal Blaschke product & mapping properties \\
\hline $\mathcal{C}=\emptyset$ & automorphism of $\mathbb{D}$ & $\begin{array}{l}\text { unbranched covering of } \mathbb{D} ; \\
\text { conformal self-map of } \mathbb{D}\end{array}$ \\
\hline $\mathcal{C}$ finite & finite Blaschke product & finite branched covering of $\mathbb{D}$ \\
\hline $\mathcal{C}$ infinite & $\begin{array}{l}\text { indestructible infinite maximal } \\
\text { Blaschke product }\end{array}$ & $\begin{array}{l}\text { "infinite branched covering of } \\
\mathbb{D} \text { " }\end{array}$ \\
\hline
\end{tabular}

The class of maximal conformal pseudometrics and their corresponding maximal functions have already been studied by Heins in [27, §25 \& §26]. Heins obtained some necessary conditions as well as sufficient conditions for maximal functions (see Theorem $\mathrm{G}$ and Theorem $\mathrm{H}$ below), but he did not prove that maximal functions are always Blaschke products. He also posed the problem of characterizing maximal functions, cf. [27, $\S 26 \& \S 29]$.

\section{Some properties of maximal Blaschke products}

It turns out that maximal Blaschke products do have remarkable properties and provide in some sense a fairly natural generalization of the class of finite Blaschke products. In this section we take a closer look at some of the properties of maximal Blaschke products. In the following, we denote by $H^{\infty}$ the set of bounded analytic functions $f: \mathbb{D} \rightarrow \mathbb{C}$ and set $\|f\|_{\infty}=\sup _{z \in \mathbb{D}}|f(z)|$. This makes $\left(H^{\infty},\|\cdot\|_{\infty}\right)$ a Banach space.

\subsection{Schwarz' lemmas.}

Theorem 3.1 (Maximal Blaschke products as extremal functions). Suppose $\mathcal{C}$ is a sequence of points in $\mathbb{D}$ such that

$$
\mathcal{F}_{\mathcal{C}}:=\left\{f \in H^{\infty}: f^{\prime}(z)=0 \text { for } z \in \mathcal{C}\right\}
$$

contains at least some non-constant function and let

$$
m:=\min \left\{n \in \mathbb{N}: f^{(n)}(0) \neq 0 \text { for some } f \in \mathcal{F}_{\mathcal{C}}\right\} \geq 1 .
$$

Then the unique extremal function to the extremal problem

$$
\max \left\{\operatorname{Re} f^{(m)}(0): f \in \mathcal{F}_{\mathcal{C}},\|f\|_{\infty} \leq 1\right\}
$$

is the maximal Blaschke product $F$ for $\mathcal{C}$ normalized by $F(0)=0$ and $F^{(m)}(0)>0$.

We refer to [44 for the proof of Theorem 3.1. To put Theorem 3.1 in perspective, note that if $m=1$, then the extremal problem (3.1) is exactly the problem of maximizing the derivative at a point, i.e., exactly the character of Schwarz' lemma.

Remark 3.2 (The Nehari-Schwarz Lemma). If $\mathcal{C}=\emptyset$, then $\mathcal{F}_{\mathcal{C}}=H^{\infty}$, i.e., the set of all bounded analytic functions in $\mathbb{D}$. In this case, Theoren 3.1 is of course just the statement of Schwarz' lemma. If $\mathcal{C}$ is a finite sequence, then Theorem 3.1 is exactly Nehari's 1947 generalization of Schwarz' lemma (Nehari [53, in particular the Corollary to Theorem 1). Hence Theorem 3.1 can be considered as an extension of the Nehari-Schwarz Lemma. 
REMARK 3.3 (The Riemann mapping theorem and the Ahlfors map). We consider a domain $\Omega \subseteq \mathbb{C}$ containing 0 . Let $\mathcal{C}=\left(z_{j}\right)$ be the critical set of a non-constant function $f$ in $H^{\infty}(\Omega)$, where $H^{\infty}(\Omega)$ denotes the set of all functions analytic and bounded in $\Omega$. We let $N$ denote the number of times that 0 appears in the sequence $\mathcal{C}$ and set

$$
\mathcal{F}_{\mathcal{C}}(\Omega):=\left\{f \in H^{\infty}(\Omega): f^{\prime}(z)=0 \text { for any } z \in \mathcal{C}\right\} .
$$

Then, by a normal family argument, there is always at least one extremal function for the extremal problem

$$
\max \left\{\operatorname{Re} f^{(N+1)}(0): f \in \mathcal{F}_{\mathcal{C}}(\Omega),\|f\|_{\infty} \leq 1\right\} .
$$

In the following three cases there is a unique extremal function to the extremal problem $(*)$.

(i) $\Omega \neq \mathbb{C}$ is simply connected and $\mathcal{C}=\emptyset$ (conformal maps):

In this case, $N=0$ and the extremal problem $(*)$ has exactly one extremal function, the normalized Riemann map $\Psi$ for $\Omega$, that is, the unique conformal map $\Psi$ from $\Omega$ onto $\mathbb{D}$ normalized such that $\Psi(0)=0$ and $\Psi^{\prime}(0)>0$.

(ii) $\Omega \neq \mathbb{C}$ is simply connected and $\mathcal{C} \neq \emptyset$ (prescribed critical points):

Let $\Psi$ be the normalized Riemann map for $\Omega$. Then $\Psi(\mathcal{C})$ is the critical set of a non-constant function in $H^{\infty}=H^{\infty}(\mathbb{D})$. If $B_{\Psi(\mathcal{C})}$ is the extremal function in $\mathcal{F}_{\Psi(\mathcal{C})}$ according to Theorem 3.1, then $B_{\Psi(\mathcal{C})} \circ \Psi$ is the unique extremal function for $(*)$.

(iii) $\Omega \neq \mathbb{C}$ is not simply connected and $\mathcal{C}=\emptyset$ (Ahlfors' maps):

If $\Omega$ has connectivity $n \geq 2$, none of whose boundary components reduces to a point, then the extremal problem $(*)$ has exactly one solution, namely the Ahlfors map $\Psi: \Omega \rightarrow \mathbb{D}$. It is a $n: 1$ map from $\Omega$ onto $\mathbb{D}$ such that $\Psi(0)=0$ and $\Psi^{\prime}(0)>0$, see Ahlfors $[2$ and Grunsky [23].

The Schwarz lemma (i.e., the case $\mathcal{C}=\emptyset$ of Theorem 3.1) can be stated in an invariant form, the Schwarz-Pick lemma which says that

$$
\frac{\left|f^{\prime}(z)\right|}{1-|f(z)|^{2}} \leq \frac{1}{1-|z|^{2}}, \quad z \in \mathbb{D}
$$

for any analytic map $f: \mathbb{D} \rightarrow \mathbb{D}$, with equality for some point $z \in \mathbb{D}$ if and only if $f$ is a conformal disk automorphism. Hence maximal Blaschke products without critical points serve as extremal functions. In a similar way, the more general statement of Theorem 3.1 admits an invariant formulation as follows (see [40]).

Theorem 3.4 (Sharpened Schwarz-Pick inequality). Let $f: \mathbb{D} \rightarrow \mathbb{D}$ be a nonconstant analytic function with critical set $\mathcal{C}$ and let $\mathcal{C}^{*}$ be a subsequence of $\mathcal{C}$. Then there exists a maximal Blaschke product $F$ with critical set $\mathcal{C}^{*}$ such that

$$
\frac{\left|f^{\prime}(z)\right|}{1-|f(z)|^{2}} \leq \frac{\left|F^{\prime}(z)\right|}{1-|F(z)|^{2}}, \quad z \in \mathbb{D}
$$

If $\mathcal{C}^{*}$ is finite, then $F$ is a finite Blaschke product.

Furthermore, $f=T \circ F$ for some automorphism $T$ of $\mathbb{D}$ if and only if

$$
\lim _{z \rightarrow w} \frac{\left|f^{\prime}(z)\right|}{1-|f(z)|^{2}} \frac{1-|F(z)|^{2}}{\left|F^{\prime}(z)\right|}=1
$$

for some $w \in \mathbb{D}$. 
If $\mathcal{C}^{*}=\mathcal{C} \neq \emptyset$, this gives the sharpening

$$
\frac{\left|f^{\prime}(z)\right|}{1-|f(z)|^{2}} \leq \frac{\left|F^{\prime}(z)\right|}{1-|F(z)|^{2}}<\frac{1}{1-|z|^{2}}
$$

for all $f \in \mathcal{F}_{\mathcal{C}}$ of the Schwarz-Pick inequality (3.2), which is best possible in some sense.

3.2. Related extremal problems in Hardy and Bergman spaces. Let $\mathcal{C}$ be a sequence in $\mathbb{D}$, assume that $\mathcal{C}$ is the critical set of a bounded analytic function $f: \mathbb{D} \rightarrow \mathbb{D}$ and let $N$ denote the multiplicity of the point 0 in $\mathcal{C}$. Then according to Theorem 3.1 the maximal Blaschke product $F$ for $\mathcal{C}$ normalized by $F(0)=0$ and $F^{(N+1)}(0)>0$ is the unique solution to the extremal problem

$$
\max \left\{\operatorname{Re} f^{(N+1)}(0): f \in H^{\infty},\|f\|_{\infty} \leq 1 \text { and } f^{\prime}(z)=0 \text { for } z \in \mathcal{C}\right\} .
$$

This extremal property of a maximal Blaschke product is reminiscent of the well-known extremal property of

(i) Blaschke products in the Hardy spaces $H^{\infty}$ and

$$
H^{p}:=\left\{f: \mathbb{D} \rightarrow \mathbb{C} \text { analytic }:\|f\|_{p}<+\infty\right\},
$$

where $1 \leq p<+\infty$ and

$$
\|f\|_{p}:=\left(\lim _{r \rightarrow 1} \frac{1}{2 \pi} \int_{0}^{2 \pi}\left|f\left(r e^{i t}\right)\right|^{p} d t\right)^{1 / p}
$$

and

(ii) canonical divisors in the (weighted) Bergman spaces

$$
\mathcal{A}_{\alpha}^{p}=\left\{f: \mathbb{D} \rightarrow \mathbb{C} \text { analytic }:\|f\|_{p, \alpha}<+\infty\right\},
$$

where $-1<\alpha<+\infty$ and $1 \leq p<+\infty$ and

$$
\|f\|_{p, \alpha}:=\left(\frac{1}{\pi} \iint_{\mathbb{D}}\left(1-|z|^{2}\right)^{\alpha}|f(z)|^{p} d \sigma_{z}\right)^{1 / p} .
$$

Note that in (i) and (ii) the prescribed data are not the critical points, but the zeros.

More precisely, let the sequence $\mathcal{C}=\left(z_{j}\right)$ in $\mathbb{D}$ be the zero set of an $H^{p}$ function and let $N$ be the multiplicity of the point 0 in $\mathcal{C}$. Then the (unique) solution to the extremal problem

$$
\max \left\{\operatorname{Re} f^{(N)}(0): f \in H^{p},\|f\|_{p} \leq 1 \text { and } f(z)=0 \text { for } z \in \mathcal{C}\right\}
$$

is a Blaschke product $B$ with zero set $\mathcal{C}$ which is normalized by $B^{(N)}(0)>0$, see [19, $§ 5.1]$.

In searching for an analogue of Blaschke products for Bergman spaces, Hedenmalm [24] (see also [17, 18] ) had the idea of posing an appropriate counterpart of the latter extremal problem for Bergman spaces. As before, let $\mathcal{C}=\left(z_{j}\right)$ be a sequence in $\mathbb{D}$ where the point 0 occurs $N$ times and assume that $\mathcal{C}$ is the zero set of a function in $\mathcal{A}_{\alpha}^{p}$. Then the extremal problem 


$$
\max \left\{\operatorname{Re} f^{(N)}(0): f \in \mathcal{A}_{\alpha}^{p},\|f\|_{p, \alpha} \leq 1 \text { and } f(z)=0 \text { for } z \in \mathcal{C}\right\}
$$

has a unique extremal function $\mathcal{G} \in \mathcal{A}_{\alpha}^{p}$, which vanishes precisely on $\mathcal{C}$ and is normalized by $\mathcal{G}^{(N)}(0)>0$. The function $\mathcal{G}$ is called the canonical divisor for $\mathcal{C}$. It plays a prominent rôle in the modern theory of Bergman spaces.

In summary, we have the following situation:

\begin{tabular}{|l|c|l|}
\hline prescribed data & function space & extremal function \\
\hline critical set $\mathcal{C}$ & $H^{\infty}$ & maximal Blaschke product for $\mathcal{C}$ \\
\hline zero set $\mathcal{C}$ & $H^{p}$ & Blaschke product \\
\hline zero set $\mathcal{C}$ & $\mathcal{A}_{\alpha}^{p}$ & canonical divisor for $\mathcal{C}$ \\
\hline
\end{tabular}

In light of this strong analogy, one expects that maximal Blaschke products enjoy similar properties as finite Blaschke products and canonical divisors. An example is their analytic continuability. It is a familiar result that a Blaschke product has a holomorphic extension across every open arc of $\partial \mathbb{D}$ that does not contain any limit point of its zero set, see [22, Chapter II, Theorem 6.1]. The same is true for a canonical divisor in the Bergman spaces $\mathcal{A}_{0}^{p}$. This was proved by Sundberg [64] in 1997, who improved earlier work of Duren, Khavinson, Shapiro and Sundberg [17, 18 and Duren, Khavinson and Shapiro [16. Now following the model that critical points of maximal functions correspond to the zeros of Blaschke products and canonical divisors respectively, one hopes that a maximal Blaschke product has an analytic continuation across every open arc of $\partial \mathbb{D}$ which does not meet any limit point of its critical set. This in fact turns out to be true:

Theorem 3.5 (Analytic continuability, [4]). Let $F: \mathbb{D} \rightarrow \mathbb{D}$ be a maximal Blaschke product with critical set $\mathcal{C}$. Then $F$ has an analytic continuation across each arc of $\partial \mathbb{D}$ which is free of limit points of $\mathcal{C}$. In particular, the limit points of the critical set of $F$ coincide with the limit points of the zero set of $F$.

Another rather strong property of finite Blaschke products is their semigroup property with respect to composition. In contrast, the composition of two infinite Blaschke products does not need to be a Blaschke product (just consider destructible Blaschke products). However, in the case of maximal Blaschke products the following result holds.

Theorem 3.6 (Semigroup property, 44]). The set of maximal Blaschke products is closed under composition.

It would be interesting to get some information about the critical values of maximal Blaschke products and to explore the possibility of factorizing maximal Blaschke products in a way similar to the recent extension of Ritt's theorem for finite Blaschke products due to $\mathrm{Ng}$ and Wang (see [52]).

3.3. Boundary behaviour of maximal Blaschke products. We now shift attention to the boundary behaviour of maximal Blaschke products. Ideally, one should be able to determine whether a bounded analytic function $F: \mathbb{D} \rightarrow \mathbb{D}$ is a maximal Blaschke produkt either from the behaviour of

$$
\frac{\left|F^{\prime}(z)\right|}{1-|F(z)|^{2}} \quad \text { as }|z| \rightarrow 1
$$


or from the behaviour of

$$
\int_{0}^{2 \pi} \log \frac{\left|F^{\prime}\left(r e^{i t}\right)\right|}{1-\left|F\left(r e^{i t}\right)\right|^{2}} d t \quad \text { as } r \rightarrow 1 .
$$

We only have some partial results in this connection and we begin our account with the case of finite Blaschke products.

THEOREM 3.7 (Boundary behaviour of finite Blaschke products). Let $I \subset \partial \mathbb{D}$ be some open arc and let $f: \mathbb{D} \rightarrow \mathbb{D}$ be an analytic function. Then the following statements are equivalent.

(a) $\lim _{z \rightarrow \zeta}\left(1-|z|^{2}\right) \frac{\left|f^{\prime}(z)\right|}{1-|f(z)|^{2}}=1 \quad$ for every $\zeta \in I$,

(b) $\lim _{z \rightarrow \zeta} \frac{\left|f^{\prime}(z)\right|}{1-|f(z)|^{2}}=+\infty \quad$ for every $\zeta \in I$,

(c) $f$ has a holomorphic extension across the arc $I$ with $f(I) \subset \partial \mathbb{D}$.

In particular, if $I=\partial \mathbb{D}$, then $f$ is in either case a finite Blaschke product.

The equivalence of conditions (a) and (b) in Theorem 3.7 for the special case $I=\partial \mathbb{D}$ is due to Heins $[\mathbf{2 8}$; the general case is proved in [41. We now extend Theorem 3.7 beyond the class of finite Blaschke products and start with the following auxiliary result.

Proposition 3.8 (see [40]). Let $f: \mathbb{D} \rightarrow \mathbb{D}$ be an analytic function and I some subset of $\partial \mathbb{D}$.

(1) If

$$
\angle \lim _{z \rightarrow \zeta}\left(1-|z|^{2}\right) \frac{\left|f^{\prime}(z)\right|}{1-|f(z)|^{2}}=1 \quad \text { for every } \zeta \in I,
$$

then $f$ has a finite angular derivative 2 at a.e. $\zeta \in I$. In particular,

$$
\angle \lim _{z \rightarrow \zeta}|f(z)|=1 \quad \text { for a.e. } \zeta \in I .
$$

(2) If $f$ has a finite angular derivative (and $\angle \lim _{z \rightarrow \zeta}|f(z)|=1$ ) at some $\zeta \in I$, then

$$
\angle \lim _{z \rightarrow \zeta}\left(1-|z|^{2}\right) \frac{\left|f^{\prime}(z)\right|}{1-|f(z)|^{2}}=1 .
$$

In particular, when $I=\partial \mathbb{D}$, we obtain the following corollary.

Corollary 3.9 (see $[40$ ). Let $f: \mathbb{D} \rightarrow \mathbb{D}$ be an analytic function. Then the following statements are equivalent.

(a) $\angle \lim _{z \rightarrow \zeta}\left(1-|z|^{2}\right) \frac{\left|f^{\prime}(z)\right|}{1-|f(z)|^{2}}=1$ for a.e. $\zeta \in \partial \mathbb{D}$.

(b) $f$ is an inner function with finite angular derivative at almost every point of $\partial \mathbb{D}$.

We further note that conditions (1) and (2) in Proposition 3.8 do not complement each other. Therefore we may ask if an analytic self-map $f$ of $\mathbb{D}$ which satisfies

$$
\angle \lim _{z \rightarrow 1} \frac{\left|f^{\prime}(z)\right|}{1-|f(z)|^{2}}\left(1-|z|^{2}\right)=1
$$

\footnotetext{
$2_{\text {see }} 60$ p. 57$]$.
} 
does have an angular limit or even a finite angular derivative at $z=1$; this might then be viewed as a converse of the Julia-Wolff-Carathéodory theorem, see [60, p. 57].

For maximal Blaschke products whose critical sets satisfy the Blaschke condition one can show that condition (a) in Corollary 3.9 holds:

Theorem 3.10 (see [39]). Let $\mathcal{C}=\left(z_{j}\right)$ be a Blaschke sequence in $\mathbb{D}$.

(a) The maximal conformal pseudometric $\lambda_{\max }(z)|d z|$ on $\mathbb{D}$ with constant curvature -4 and zero set $\mathcal{C}$ satisfies

$$
\angle \lim _{z \rightarrow \zeta} \frac{\lambda_{\max }(z)}{\lambda_{\mathbb{D}}(z)}=1 \quad \text { for a.e. } \zeta \in \partial \mathbb{D} .
$$

(b) Every maximal function for $\mathcal{C}$ has a finite angular derivative at almost every point of $\partial \mathbb{D}$.

REMARK 3.11. The boundary behaviour of a maximal Blaschke product contains useful information. For instance, it leads to a quick proof of Theorem F To see this, let $F: \mathbb{D} \rightarrow \mathbb{D}$ be a maximal function for a finite sequence $\mathcal{C}$ and $\lambda_{\max }(z)|d z|=\left(F^{*} \lambda_{\mathbb{D}}\right)(z)|d z|$ be the maximal conformal metric with constant curvature -4 and zero set $\mathcal{C}$. By Theorem 2.9, the maximal function $F$ is an indestructible Blaschke product. Now, let $B$ be a finite Blaschke product with zero set C. Then

$$
\lambda(z)|d z|:=|B(z)| \lambda_{\mathbb{D}}(z)|d z|
$$

is a regular conformal pseudometric on $\mathbb{D}$ with curvature $-4 /|B(z)|^{2} \leq-4$ and zero set $\mathcal{C}$. Thus, by the maximality of $\lambda_{\max }(z)|d z|$,

$$
\lambda(z) \leq \lambda_{\max }(z) \quad \text { for } z \in \mathbb{D}
$$

and consequently

$$
|B(z)| \leq \frac{\lambda_{\max }(z)}{\lambda_{\mathbb{D}}(z)} \quad \text { for all } z \in \mathbb{D} .
$$

Since $B$ is a finite Blaschke product, we deduce from (3.3) and the Schwarz-Pick lemma (3.2) that

$$
\lim _{z \rightarrow \zeta} \frac{\lambda_{\max }(z)}{\lambda_{\mathbb{D}}(z)}=\lim _{z \rightarrow \zeta} \frac{\left|F^{\prime}(z)\right|}{1-|F(z)|^{2}}\left(1-|z|^{2}\right)=1 \quad \text { for all } \zeta \in \partial \mathbb{D} .
$$

Applying Theorem 3.7 shows that $F$ is a finite Blaschke product. The branching order of $F$ is clearly $2 n$. Thus, according to the Riemann-Hurwitz formula, see [21. p. 140], the Blaschke product $F$ has degree $m=n+1$.

On the other hand, assume that $F$ is a finite Blaschke product of degree $n+1$. Then, by Theorem 3.7 .

$$
\lim _{z \rightarrow \zeta} \frac{\left|F^{\prime}(z)\right|}{1-|F(z)|^{2}}\left(1-|z|^{2}\right)=1 \quad \text { for all } \zeta \in \partial \mathbb{D} .
$$

Theorem 3.12 below shows that $F$ is a maximal Blaschke product.

The next result gives a sufficient condition for maximality of a Blaschke product $F$ in terms of the boundary behaviour of the integral means of the quantity

$$
\left(1-|z|^{2}\right) \frac{\left|F^{\prime}(z)\right|}{1-|F(z)|^{2}}=\frac{\lambda(z)}{\lambda_{\mathbb{D}}(z)} .
$$


THEOREM 3.12 (see [39]). Let $\lambda(z)|d z|$ be a conformal pseudometric on $\mathbb{D}$ with constant curvature -4 and zero set $\mathcal{C}$ such that

$$
\lim _{r \rightarrow 1} \int_{0}^{2 \pi} \log \frac{\lambda\left(r e^{i t}\right)}{\lambda_{\mathbb{D}}\left(r e^{i t}\right)} d t=0 .
$$

Then $\lambda(z)|d z|$ is the maximal conformal pseudometric $\lambda_{\max }(z)|d z|$ on $\mathbb{D}$ with constant curvature -4 and zero set $\mathcal{C}$.

If $\mathcal{C}$ is a Blaschke sequence, then the corresponding maximal conformal pseudometric $\lambda_{\max }(z)|d z|$ on $\mathbb{D}$ with constant curvature -4 and zero set $\mathcal{C}$ satisfies condition (3.4):

Theorem 3.13 (see 40 ). Let $\mathcal{C}$ be a Blaschke sequence in $\mathbb{D}$. A conformal pseudometric $\lambda(z)|d z|$ on $\mathbb{D}$ with constant curvature -4 and zero set $\mathcal{C}$ is the maximal conformal pseudometric $\lambda_{\max }(z)|d z|$ on $\mathbb{D}$ with constant curvature -4 and zero set $\mathcal{C}$ if and only if (3.4) holds.

We don't know whether this result is true for any sequence $\mathcal{C}$ for which there is a non-constant bounded analytic function with critical set $\mathcal{C}$.

3.4. Heins' results on maximal functions. For completeness, we close this section with a discussion of Heins' results on maximal functions, cf. [27, §25 \& §26]. A first observation is that every maximal function is surjective. In fact more is true; a maximal function is "locally" surjective. Here is the precise definition.

Definition 3.14. Let $f: \mathbb{D} \rightarrow \mathbb{D}$ be an analytic function. A point $q \in \mathbb{D}$ is called locally omitted by $f$ provided that either $q \in \mathbb{D} \backslash f(\mathbb{D})$ or else $q \in f(\mathbb{D})$ and there exists a domain $\Omega, q \in \Omega$, such that for some component $U$ of $f^{-1}(\Omega)$ the restriction of $f$ to $U$ omits $q$, i.e. $q \notin f(U)$.

TheOREM G (Heins [27]). A maximal function has no locally omitted point.

So far, the results about maximal functions leave an important question unanswered, namely, how to tell whether a given function in $H^{\infty}$ is a maximal function? Heins' second result gives a topological sufficient criterion and provides therefore a source of "examples" of maximal functions. It is based on the following concept.

Definition 3.15. A holomorphic function $f: \mathbb{D} \rightarrow \mathbb{D}$ is called locally of island type if $f$ is onto and if for each $w \in \mathbb{D}$ there is an open disk $K(w)$ about $w$ such that each component of $f^{-1}(K(w))$ is compactly contained in $\mathbb{D}$.

Obviously, every surjective analytic self-map of $\mathbb{D}$ with constant finite valence, that is, every finite Blaschke product is locally of island type.

THEOREM H (Heins [27]). Every function locally of island type is a maximal function.

\section{The Gauss curvature PDE and the Berger-Nirenberg problem}

We return to a discussion of Theorem 1.1. The results of Section 2 (Theorem E and Theorem 2.9) provide a proof of implication "(a) $\Longrightarrow(\mathrm{b})$ " in Theorem 1.1 In this section, we discuss implication "(c) $\Longrightarrow(\mathrm{a})$ ". The key idea is the following.

THEOREM 4.1. Let $h: \mathbb{D} \rightarrow \mathbb{C}$ be a non-constant holomorphic function with zero set $\mathcal{C}$. Then the following statements are equivalent. 
(a) There exists a holomorphic function $f: \mathbb{D} \rightarrow \mathbb{D}$ with critical set $\mathcal{C}$.

(b) There exists a $C^{2}$-solution $u: \mathbb{D} \rightarrow \mathbb{R}$ to the Gauss curvature equation

$$
\Delta u=4|h(z)|^{2} e^{2 u} .
$$

Let us sketch a proof here. If $f: \mathbb{D} \rightarrow \mathbb{D}$ is a holomorphic function with critical set $\mathcal{C}$, then a quick computation shows that

$$
u(z):=\log \left(\frac{1}{|h(z)|} \frac{\left|f^{\prime}(z)\right|}{1-|f(z)|^{2}}\right)
$$

is a $C^{2}$-solution to (4.1). This proves "(a) $\Longrightarrow(\mathrm{b})$ ". Conversely, if there is a $C^{2}$-solution $u: \mathbb{D} \rightarrow \mathbb{R}$ to the curvature equation (4.1), then

$$
\lambda(z)|d z|:=e^{u(z)}|d z|
$$

is a regular conformal metric with curvature $-4|h(z)|^{2}$ on $\mathbb{D}$. Hence, by Theorem 2.5 .

$$
u(z)=\log \left(\frac{1}{|h(z)|} \frac{\left|f^{\prime}(z)\right|}{1-|f(z)|^{2}}\right)
$$

with some analytic self-map $f$ of $\mathbb{D}$. Thus the zero set of $h$ agrees with the critical set of $f$.

In view of Theorem 4.1, the task is now to characterize those holomorphic functions $h: \mathbb{D} \rightarrow \mathbb{C}$ for which the PDE (4.1) has a solution. In fact this problem is a special case of the Berger-Nirenberg problem from differential geometry:

\section{Berger-Nirenberg problem:}

Given a function $\kappa: R \rightarrow \mathbb{R}$ on a Riemann surface $R$. Is there a conformal metric on $R$ with Gauss curvature $\kappa$ ?

The Berger-Nirenberg problem is well-understood for the projective plane, see 51 and has been extensively studied for compact Riemannian surfaces, see 3, 9, 33, 63, as well as for the complex plane [4, 10, 55 3. However much less is known for proper domains $D$ of the complex plane, see [6, 31, 34. In this situation the Berger-Nirenberg problem reduces to the question if for a given function $k: D \rightarrow \mathbb{R}$ the Gauss curvature equation

$$
\Delta u=k(z) e^{2 u}
$$

has a solution on $D$. We just note that $k$ is the negative of the curvature $\kappa$ of the conformal metric $e^{u(z)}|d z|$.

In the next theorem we give some necessary conditions as well as sufficient conditions for the solvability of the Gauss curvature equation (4.2) only in terms of the curvature function $k$ and the domain $D$.

TheOREM 4.2 (see [40]). Let $D$ be a bounded and regular domain and let $k$ be a nonnegative locally Hölder continuous function on $D$.

(1) If for some (and therefore for every) $z_{0} \in D$

$$
\iint_{D} g_{D}\left(z_{0}, \xi\right) k(\xi) d \sigma_{\xi}<+\infty,
$$

then (4.2) has a $C^{2}$-solution $u: D \rightarrow \mathbb{R}$, which is bounded from above.

\footnotetext{
${ }^{3}$ These are just some of the many references.

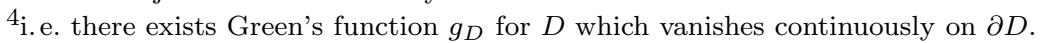


(2) If (4.2) has a $C^{2}$-solution $u: D \rightarrow \mathbb{R}$ which is bounded from below and if this solution has a harmonic majorant on $D$, then

$$
\iint_{D} g_{D}(z, \xi) k(\xi) d \sigma_{\xi}<+\infty
$$

for all $z \in D$.

(3) There exists a bounded $C^{2}$-solution $u: D \rightarrow \mathbb{R}$ to 4.2) if and only if

$$
\sup _{z \in D} \iint_{D} g_{D}(z, \xi) k(\xi) d \sigma_{\xi}<+\infty
$$

If we choose $D=\mathbb{D}$ and $z_{0}=0$, then $g_{\mathbb{D}}(0, \xi)=-\log |\xi|$. Hence as a consequence of the inequality

$$
\frac{1-|\xi|^{2}}{2} \leq \log \frac{1}{|\xi|} \leq \frac{1-|\xi|^{2}}{|\xi|}, \quad 0<|\xi|<1
$$

we obtain the following equivalent formulation of Theorem 4.2

Corollary 4.3 (see 40 ). Let $k$ be a nonnegative locally Hölder continuous function on $\mathbb{D}$.

(1) If

$$
\iint_{\mathbb{D}}\left(1-|\xi|^{2}\right) k(\xi) d \sigma_{\xi}<+\infty
$$

then (4.2) has a $C^{2}$-solution $u: \mathbb{D} \rightarrow \mathbb{R}$, which is bounded from above.

(2) If (4.2) has a $C^{2}$-solution $u: \mathbb{D} \rightarrow \mathbb{R}$ which is bounded from below and if this solution has a harmonic majorant on $\mathbb{D}$, then

$$
\iint_{\mathbb{D}}\left(1-|\xi|^{2}\right) k(\xi) d \sigma_{\xi}<+\infty .
$$

(3) There exists a bounded $C^{2}$-solution $u: \mathbb{D} \rightarrow \mathbb{R}$ to 4.2) if and only if

$$
\sup _{z \in \mathbb{D}} \iint_{\mathbb{D}} \log \left|\frac{1-\bar{\xi} z}{z-\xi}\right| k(\xi) d \sigma_{\xi}<+\infty .
$$

Both, Theorem 4.2 and Corollary 4.3, are not best possible, because (4.2) may indeed have solutions, even if

$$
\iint_{D} g_{D}(z, \xi) k(\xi) d \sigma_{\xi}=+\infty
$$

for some (and therefore for all) $z \in D$. Here is an explicit example.

ExAmPLE 4.4. For $\alpha \geq 3 / 2$ define

$$
h(z)=\frac{1}{(z-1)^{\alpha}}
$$

for $z \in \mathbb{D}$ and set $k(z)=4|h(z)|^{2}$ for $z \in \mathbb{D}$. Then an easy computation yields

$$
\iint_{\mathbb{D}}\left(1-|z|^{2}\right) k(z) d \sigma_{z}=+\infty
$$


and a straightforward check shows that the function

$$
u_{f}(z):=\log \left(\frac{1}{|h(z)|} \frac{\left|f^{\prime}(z)\right|}{1-|f(z)|^{2}}\right)
$$

is a solution to (4.2) on $\mathbb{D}$ for every locally univalent analytic function $f: \mathbb{D} \rightarrow \mathbb{D}$.

REMARK 4.5. The sufficient condition (4.3) improves earlier results of Kalka \& Yang in 34. In fact, Kalka \& Yang give explicit examples for the function $k$ which tend to $+\infty$ at the boundary of $\mathbb{D}$ such that the existence of a solution to (4.2) can be guaranteed. All these examples are radially symmetric and satisfy (4.3). Kalka \& Yang also supplement their existence results by nonexistence results. They find explicit lower bounds for the function $k$ in terms of radially symmetric functions which grow to $+\infty$ at the boundary of $\mathbb{D}$, such that (4.2) has no solution.

We wish to emphasize that the necessary conditions and the sufficient conditions for the solvability of the curvature equation (4.2) of Kalka \& Yang do not complement each other. In particular, the case when the function $k$ oscillates is not covered. For the proof of their nonexistence results Kalka \& Yang needed to use Yau's celebrated Maximum Principle [68, 69], which is an extremely powerful tool. In [39], an almost elementary proof of these nonexistence results is given, which has the additional advantage that Ahlfors' type lemmas for conformal metrics with variable curvature and explicit formulas for the corresponding maximal conformal metrics are obtained. In [39] the nonexistence theorems of Kalka \& Yang are further extended by allowing the function $k$ to oscillate.

It turns out that although condition (4.3) is not necessary for the existence of a solution to (4.2) it is strong enough to deduce a necessary and sufficient condition for the solvability of the Gauss curvature equation of the particular form (4.1):

ThEOREM 4.6 (see 40 ). Let $h: \mathbb{D} \rightarrow \mathbb{C}$ be a holomorphic function. Then the Gauss curvature equation (4.1) has a solution if and only if $h$ has a representation as a product of an $\mathcal{A}_{1}^{2}$ function and a nonvanishing analytic function.

Note that Theorem 4.1 combined with Theorem 4.6 shows that the class of all holomorphic functions $h: \mathbb{D} \rightarrow \mathbb{C}$ whose zero sets coincide with the critical sets of the class of bounded analytic function is exactly the Bergman space $\mathcal{A}_{1}^{2}$. This proves implication "(c) $\Longrightarrow(\mathrm{a})$ " in Theorem 1.1

A further remark is that Theorem 4.2 (c) characterizes those curvature functions $k$ for which (4.2) has at least one bounded solution. For the case of the unit disk $\mathbb{D}$, this result can be stated as follows.

TheOREM 4.7. Let $\varphi: \mathbb{D} \rightarrow \mathbb{C}$ be analytic and $k(z)=4\left|\varphi^{\prime}(z)\right|^{2}$. Then there exists a bounded solution to the Gauss curvature equation (4.2) if and only if $\varphi \in$ $B M O A$, where

$$
B M O A=\left\{\varphi: \mathbb{D} \rightarrow \mathbb{C} \text { analytic }: \sup _{z \in \mathbb{D}} \iint_{\mathbb{D}} g_{\mathbb{D}}(z, \xi)\left|\varphi^{\prime}(\xi)\right|^{2} d \sigma_{\xi}<+\infty\right\}
$$

is the space of analytic functions of bounded mean oscillation on $\mathbb{D}$, see [45, p. 314].

Finally, we note that in Theorem 4.2 and Corollary 4.3 , condition (1) does not imply condition (3). The Gauss curvature equation (4.2) may indeed have solutions none of which is bounded. For example, choose $\varphi \in H^{2} \backslash B M O A$ and set 
$k(z)=4\left|\varphi^{\prime}(z)\right|^{2}$. Then, according to Theorem 4.7, every solution to (4.2) must be unbounded.

The following result of Heins adds another item to the list of equivalent statements in Theorem 1.1.

Theorem I (Heins [27). Let $\mathcal{C}=\left(z_{j}\right)$ be a sequence in $\mathbb{D}$. Then the following conditions are equivalent.

(a) There is an analytic function $f: \mathbb{D} \rightarrow \mathbb{D}$ with critical set $\left(z_{j}\right)$.

(b) There is a function in the Nevanlinna class $\mathcal{N}$ with critical set $\left(z_{j}\right)$.

Here, a function $f$ analytic in $\mathbb{D}$ is said to belong to the Nevanlinna class $\mathcal{N}$ if the integrals

$$
\int_{0}^{2 \pi} \log ^{+}\left|f\left(r e^{i t}\right)\right| d t
$$

remain bounded as $r \rightarrow 1$.

Let us indicate how the results of the present survey allow a quick proof of Theorem [1

Proof. (b) $\Rightarrow$ (a): Let $\varphi \in \mathcal{N}$. Then $\varphi=\varphi_{1} / \varphi_{2}$ is the quotient of two analytic self-maps of $\mathbb{D}$, see for instance [15, Theorem 2.1]. W.1.o.g. we may assume $\varphi_{2}$ is zerofree. Differentiation of $\varphi$ yields

$$
\varphi^{\prime}(z)=\frac{1}{\varphi_{2}(z)^{2}}\left(\varphi_{1}^{\prime}(z) \varphi_{2}(z)-\varphi_{1}(z) \varphi_{2}^{\prime}(z)\right) .
$$

Since $\varphi_{1}^{\prime}, \varphi_{2}^{\prime} \in \mathcal{A}_{1}^{2}$ and $\mathcal{A}_{1}^{2}$ is a vector space, it follows that the function $\varphi_{1}^{\prime} \varphi_{2}-\varphi_{1} \varphi_{2}^{\prime}$ belongs to $\mathcal{A}_{1}^{2}$. Thus Theorem 4.6 ensures the existence of a solution $u: \mathbb{D} \rightarrow \mathbb{R}$ to

$$
\Delta u=\left|\varphi^{\prime}(z)\right|^{2} e^{2 u} .
$$

Hence Theorem 4.1 gives the desired result.

The results of this section about the solvability of the Gauss curvature equation (4.2) do have further consequences for the critical sets of bounded analytic functions. For instance, one can give an answer to a question of Heins [27, §31]. In order to state Heins' question properly, we recall the well-known Jensen formula which connects the rate of growth of an analytic function with the density of its zeros. Thus the restriction on the growth of the derivative of an analytic self-map of $\mathbb{D}$ imposed by the Schwarz-Pick lemma (3.2) forces an upper bound for the number of critical points of non-constant analytic self-maps of $\mathbb{D}$. More precisely,

$$
\sup _{\substack{f \in H^{\infty} \\\|f\| \infty \leq 1, f \neq c \text { const. }}}\left(\limsup _{r \rightarrow 1} \frac{N\left(r ; f^{\prime}\right)}{\log \frac{1}{1-r^{2}}}\right) \leq 1,
$$

where

$$
N\left(r ; f^{\prime}\right):=\int_{0}^{1} \frac{n\left(t ; f^{\prime}\right)}{t} d t
$$

and $n\left(r ; f^{\prime}\right)$ denotes the number of zeros of $f^{\prime}$ counted with multiplicity in the disk $\mathbb{D}_{r}:=\{z \in \mathbb{C}:|z|<r\}, 0<r<1$. 
Heins showed that equality holds in (4.4), cf. [26. More precisely, using his solution of the Schwarz-Picard problem, he proved that there exists for every $p=$ $2,3, \ldots$ a non-constant analytic function $f_{p}: \mathbb{D} \rightarrow \mathbb{D}$ such that

$$
\limsup _{r \rightarrow 1} \frac{N\left(r ; f_{p}^{\prime}\right)}{\log \left(\frac{1}{1-r^{2}}\right)}=\frac{2 p-3}{2 p-2} .
$$

Letting $p \rightarrow+\infty$ shows that (4.4) is best possible. In this way, Heins [27, §31] was led to ask whether there is a bounded analytic function which realizes the supremum in (4.4). This question is answered in our next theorem - even with some additional information.

THEOREM 4.8. Let $\beta \in[0,1]$. Then there exists a non-constant analytic function (and even a maximal Blaschke product) $f: \mathbb{D} \rightarrow \mathbb{D}$ such that

$$
\limsup _{r \rightarrow 1} \frac{N\left(r ; f^{\prime}\right)}{\log \left(\frac{1}{1-r^{2}}\right)}=\beta .
$$

For the proof of Theorem 4.8 we refer the reader to $\mathbf{3 9}$.

We close with the following remark.

REMARK 4.9. With the help of the Riemann mapping theorem, the results of this paper about critical sets of bounded analytic functions $f: \mathbb{D} \rightarrow \mathbb{D}$ can easily be transferred to the class $H^{\infty}(\Omega)$ of bounded analytic functions $f: \Omega \rightarrow \mathbb{D}$, when $\Omega \neq \mathbb{C}$ is a simply connected domain. The critical sets of bounded analytic functions on multiply connected domains are much more difficult to fathom.

\section{Acknowledgement}

This paper is based on lectures given at the workshop on Blaschke products and their Applications (Fields Institute, Toronto, July 25-29, 2011). The authors would like to thank the organizers of this workshop, Javad Mashreghi and Emmanuel Fricain, as well as the Fields Institute and its staff, for their generous support and hospitality.

\section{References}

[1] L. Ahlfors, An extension of Schwarz's lemma, Trans. Am. Math. Soc. 43 (1938), 359-364.

[2] L. Ahlfors, Bounded analytic functions, Duke Math. J. 14 (1947), 1-11.

[3] T. Aubin, Some nonlinear problems in Riemannian geometry, Springer 1998.

[4] P. Aviles, Conformal complete metrics with prescribed non-negative Gaussian curvature in $\mathbb{R}^{2}$, Invent. Math. 83 (1986), 519-544.

[5] A. Beardon and D. Minda, The Hyperbolic Metric and Geometric Function Theory, in: S. Ponnusamy, T. Sugawa and M. Vuorinen (editors), Quasiconformal Mappings and their Applications, Narosa 2007.

[6] J. Bland and M. Kalka, Complete metrics conformal to the hyperbolic disc, Proc. Am. Math. Soc. 97 (1986), no. 1, 128-132.

[7] W. Blaschke, Eine Erweiterung des Satzes von Vitali über Folgen analytischer Funktionen, S.-B. Sächs. Akad. Wiss. Leipzig Math.-Natur. Kl. 67 (1915), 194-200.

[8] L. Bieberbach, $\Delta u=e^{u}$ und die automorphen Funktionen, Math. Ann. 77 (1916), 173-212.

[9] S. Y. A. Chang, Non-linear elliptic equations in conformal geometry, European Mathematical Society 2004.

[10] K. S. Cheng and W. M. Ni, On the structure of the conformal Gaussian curvature equation on $\mathbb{R}^{2}$, Duke Math.J. 62 (1991), no. 3, 721-737. 
[11] K. S. Chou and T. Wan, Asymptotic radial symmetry for solutions of $\Delta u+e^{u}=0$ in a punctured disc, Pacific J. Math. 163 (1994), no. 2, 269-276.

[12] K. S. Chou and T. Wan, Correction to "Asymptotic radial symmetry for solutions of $\Delta u+e^{u}=$ 0 in a punctured disc", Pacific J. Math. 171 (1995), no. 2, 589-590.

[13] P. Colwell, Blaschke Products, The University of Michigan Press 1985.

[14] P. Duren, On the Bloch-Nevanlinna conjecture, Colloq. Math. 20 (1969), 295-297.

[15] P. Duren, Theory of $H^{p}$ Spaces, Dover 2000.

[16] P. Duren, D. Khavinson and H. S. Shapiro, Extremal functions in invariant subspaces of Bergman spaces, Illinois J. Math. 40 (1996), 202-210.

[17] P. Duren, D. Khavinson, H. S. Shapiro and C. Sundberg, Contractive zero-divisors in Bergman spaces, Pacific J. Math. 157 (1993), no. 1, 37-56.

[18] P. Duren, D. Khavinson, H. S. Shapiro and C. Sundberg, Invariant subspaces in Bergman spaces and the biharmonic equation, Michigan Math. J. 41 (1994), no. 2, 247-259.

[19] P. Duren and A. Schuster, Bergman Spaces, Am. Math. Soc. 2004.

[20] P. Ebenfelt, D. Khavinson and H.S. Shapiro, Two-dimensional shapes and lemniscates, to appear in Complex Analysis \& Dynamical Systems IV, Contemp. Math., see also http://arxiv.org/abs/1003.4567.

[21] O. Forster, Lectures on Riemann surfaces, Springer 1999.

[22] J. B. Garnett, Bounded analytic functions, revised first edition, Springer 2007.

[23] H. Grunsky, Lectures on theory of functions in multiply connected domains, Vandenhoeck \& Rupprecht 1978.

[24] H. Hedenmalm, A factorization theorem for square area-integrable analytic functions, J. Reine Angew. Math. 442 (1991), 45-68.

[25] H. Hedenmalm, B. Korenblum and K. Zhu, Theory of Bergman Spaces, Springer 2000.

[26] M. Heins, A class of conformal metrics, Bull. Am. Math. Soc. 67 (1961), 475-478.

[27] M. Heins, On a class of conformal metrics, Nagoya Math. J. 21 (1962), 1-60.

[28] M. Heins, Some characterizations of finite Blaschke products of positive degree, J. Anal. Math. 46 (1986), 162-166.

[29] C. Horowitz, Zeros of functions in the Bergman spaces, Duke Math. J. 41 (1974), 693-710.

[30] C. Horowitz, Factorization theorems for functions in the Bergman spaces, Duke Math. J. 44 (1977), 201-213.

[31] D. Hulin and M. Troyanov, Prescribing curvature on open surfaces, Math. Ann. 293 (1992), no. $2,277-315$.

[32] J. Jensen, Sur un nouvel et important théorème de la théorie des fonctions, Acta Math. 22 (1899), 359-364.

[33] J. Kazdan, Prescribing the curvature of a Riemannian manifold, CMBS Regional Conf. Ser. in Math. 57 (1985).

[34] M. Kalka and D. Yang, On conformal deformation of nonpositive curvature on noncompact surfaces, Duke Math.J. 72 (1993), no. 2, 405-430.

[35] L. Keen and N. Lakic, Hyperbolic Geometry from a Local Viewpoint, Camb. Univ. Press 2007.

[36] P. Koosis, Introduction to $H^{p}$ Spaces, Cambridge Tracts, Second Edition, 1998.

[37] B. Korenblum, An extension of the Nevanlinna theory, Acta Math. 135 (1975), 187-219.

[38] St. Krantz, Complex Analysis - The geometric Viewpoint, Second Edition, Math. Ass. Amer., 2004.

[39] D. Kraus, Critical sets of bounded analytic functions, zero sets of Bergman spaces and nonpositive curvature, Habilitationsschrift Universität Würzburg 2010.

[40] D. Kraus, Critical sets of bounded analytic functions, zero sets of Bergman spaces and nonpositive curvature, submitted.

[41] D. Kraus, O. Roth and St. Ruscheweyh, A boundary version of Ahlfors' Lemma, locally complete conformal metrics and conformally invariant reflection principles for analytic maps, J. Anal. Math. 101 (2007), 219-256.

[42] D. Kraus and O. Roth, Critical points of inner functions, nonlinear partial differential equations, and an extension of Liouville's theorem, J. London Math. Soc. 77 (2008), no. 1, 183202.

[43] D. Kraus and O. Roth, Conformal Metrics, in: Topics in Modern Function Theory, Ramanujan Math. Soc., 41 pp., to appear.

[44] D. Kraus and O. Roth, Maximal Blaschke products, submitted. 
[45] I. Laine, Complex differential equations, in: F. Battelli and M. Fečkan (editors), Handbook of differential equations: Ordinary differential equations, Vol. IV, Elsevier 2008.

[46] J. Liouville, Sur l'équation aux différences partielles $\frac{d^{2} \log \lambda}{d u d v} \pm \frac{\lambda}{2 a^{2}}=0$, J. de Math. 16 (1853), $71-72$.

[47] D.H. Luecking, Zero sequences for Bergman spaces, Compl. Variables Theory Appl. 30 (1996), no. $4,345-362$.

[48] J. Mashreghi, Representation Theorems in Hardy Spaces, LMS Student Texts 74, 2009.

[49] C. D. Minda, The hyperbolic metric and coverings of Riemann surfaces, Pacific J. Math. 84 (1979), no. 1, 171-182.

[50] D. Minda, Conformal metrics, unpublished notes.

[51] J. Moser, On a nonlinear problem in differential geometry, Dynamical Syst., Proc. Sympos. Univ. Bahia, Salvador 1971 (1973), 273-280.

[52] T. W. Ng and M.-X. Wang, Ritt's theory on the unit disk, preprint 2011.

[53] Z. Nehari, A generalization of Schwarz' lemma, Duke Math. J. 14 (1947), 1035-1049.

[54] F. and R. Nevanlinna, Über die Eigenschaften analytischer Funktionen in der Umgebung einer singulären Stelle oder Linie, Acta Soc. Sci. Fenn. 50 (1922), no. 5, 46 pp.

[55] W.-M. Ni, Recent progress on the elliptic equation $\Delta u+K e^{2 u}=0$ on $\mathbb{R}^{2}$, Rend. Semin. Mat., Torino Fasc. Spec. 1989, 1-10.

[56] J. Nitsche, Über die isolierten Singularitäten der Lösungen von $\Delta u=e^{u}$, Math. Z. 68 (1957), $316-324$.

[57] K. Seip, On a theorem of Korenblum, Ark. Mat. 32 (1994), 237-243.

[58] K. Seip, On Korenblum's density condition for zero sequences of $A^{-\alpha}$, J. Anal. Math. 67 (1995), 307-322.

[59] H. S. Shapiro and A. L. Shields, On the zeros of functions with finite Dirichlet integral and some related function spaces, Math.Z. 80 (1962), 217-229.

[60] J. H. Shapiro, Composition operators and classical function theory, Springer 1993.

[61] S. J. Smith, On the uniformization of the n-punctured disc, Ph. D. Thesis, University of New England, 1986.

[62] K. Stephenson, Introduction to circle packing: the theory of discrete analytic functions, Camb. Univ. Press 2005.

[63] M. Struwe, A flow approach to Nirenberg's problem, Duke Math. J. 128 (2005), no. 1, 19-64.

[64] C. Sundberg, Analytic continuability of Bergman inner functions, Michigan Math.J. 44 (1997), no. 2, 399-407.

[65] J. Walsh, The location of critical points of analytic and harmonic functions, Am. Math. Soc. 1950.

[66] Q. Wang and J. Peng, On critical points of finite Blaschke products and the equation $\Delta u=$ $e^{2 u}$, Kexue Tongbao 24 (1979), 583-586 (Chinese).

[67] A. Yamada, Bounded analytic functions and metrics of constant curvature on Riemann surfaces, Kodai Math.J. 11 (1988), no. 3, 317-324.

[68] S. T. Yau, Harmonic functions on complete Riemannian manifolds, Comm. Pure Appl. Math. 28 (1975), 201-228.

[69] S. T. Yau, A general Schwarz lemma for Kähler manifolds, Am. J. Math. 100 (1978), 197-203.

[70] S. Zakeri, On critical points of proper holomorphic maps on the unit disk, Bull. London Math. Soc. 30 (1996), no. 1, 62-66.

Department of Mathematics, University of Würzburg, Emil-Fischer Strasse 40, 97074 Würzburg, Germany

E-mail address: dakraus@mathematik.uni-wuerzburg.de

Department of Mathematics, University of Würzburg, Emil-Fischer Strasse 40, 97074 WürzBurg, Germany

E-mail address: roth@mathematik.uni-wuerzburg.de 